\title{
Aqueous Photon Upconversion by Anionic Acceptors Self-Assembled on Cationic Bilayer Membranes with a Long Triplet Lifetime
}

\author{
Deepak Asthanaa,b (D) \\ Shota Hisamitsua (i) \\ Masa-aki Morikawa ${ }^{a}$ \\ Pengfei Duan ${ }^{a, c}$ (D) \\ Takuya Nakashimad (D) \\ Tsuyoshi Kawaid (iD \\ Nobuhiro Yanai*a,e (D) \\ Nobuo Kimizuka*a (D) \\ Department of Chemistry and Biochemistry, Graduate School of Engineering, Cente \\ for Molecular Systems (CMS), Kyushu University, \\ 744 Moto-oka, Nishi-ku, Fukuoka 819-0395, Japan \\ yanai@mail.cstm.kyushu-u.ac.jp \\ n-kimi@mail.cstm.kyushu-u.ac.jp \\ b School of Chemistry, The University of Manchester, Manchester, United Kingdom \\ 'National Center for Nanoscience and Technology, Zhongguancun, Beijing, China \\ Graduate School of Materials Science, Nara Institute of Science and Technology, \\ Ikoma, Nara, Japan \\ PRESTO, JST, Kawaguchi, Saitama, Japan
}

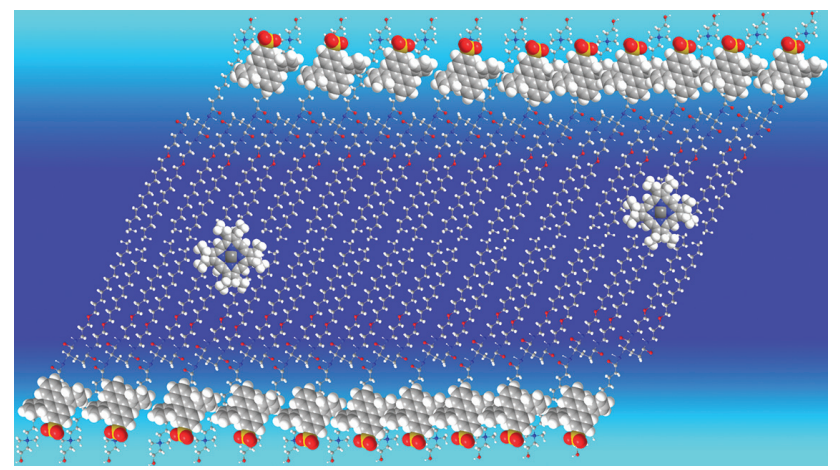

Received: 28.08.2019

Accepted after revision: 24.09.2019

DOI: 10.1055/s-0039-3400250; Art ID: om-19-0009-sc

License terms: (c)

(c) 2019. The Author(s). This is an open access article published by Thieme under the terms of the Creative Commons Attribution-NonDerivative-NonCommercial-License, permitting copying and reproduction so long as the original work is given appropriate credit. Contents may not be used for commercial purposes, or adapted, remixed, transformed or built upon. (https://creativecommons.org/licenses/by-nc-nd/4.0/).

Abstract Anionic 9,10-diphenylanthracene chromophores electrostatically bound to cationic, chiral bilayer membranes show ordered selfassembly in water. The integrity of the chromophore-accumulated aqueous bilayer membranes is ensured by multiple hydrogen-bond networks introduced in the bilayer, which allowed adaptive accommodation of the guest chromophores at the inner surface of the bilayer while maintaining their cohesive interactions. The regular chromophore alignment in the aqueous assembly is confirmed by differential scanning calorimetry, circular dichroism, and circularly polarized luminescence spectra. Excitonic migration of triplet energy occurs among the chromophores densely organized at the inner surface of the bilayer, which lead to triplet-triplet annihilation-based photon upconversion (TTA-UC). This acceptor-bilayer self-assemblies show a notably long triplet lifetime of $8.0 \mathrm{~ms}$, which allows TTA-UC at sufficiently low excitation light intensity. These results demonstrate the usefulness of the simple electrostatic accumulation approach for TTA-UC chromophores where the suitable molecular design of the TTA-UC chromophore-integrated bilayer membranes plays a key role.

Key words self-assembly, photon upconversion, triplet, triplet-triplet annihilation, bilayer membranes, energy migration

\section{Introduction}

The photosynthetic molecular systems in green plants show efficient harvesting of solar photon energy which is achieved by the antenna chlorophyll-protein complexes integrated in the thylakoid membrane. ${ }^{1,2}$ Although chlorophylls alone do not show self-assembling properties, they are adaptively organized in the host proteins which hierarchically self-assemble into the light-harvesting complexes with intermolecular distances and orientations finely regulated by the host protein arrays. Inspired by the efficient singlet energy migration in the light-harvesting complexes, self-assembly of chromophores have been investigated for a wide variety of synthetic systems with the goal of achieving excitonic interactions, efficient singlet energy migration, and energy transfer. ${ }^{3-11}$ The transfer of singlet energy mostly occurs via the Förster resonance energy transfer mechanism, which is a dissipation process of the excitation photon energy. Meanwhile, the conversion of the absorbed photon energy to higher energy in the form of a photon or chemical energy and its effective utilization are challenges of significant importance which meets the increasing demands for renewable energy generation. ${ }^{12-22}$

Based on this perspective, we developed a series of photon upconversion molecular systems that harnessed triplet energy migration (TEM) and triplet-triplet annihilation (TTA) in designed self-assemblies. ${ }^{23-28}$ The TTA-based photon upconversion (TTA-UC) has attracted considerable interest because it permits the intrinsic spectral mismatch between the solar emission and device absorption profiles to be overcome. ${ }^{12-19,23-28}$ One of the current challenges is the 
application of TTA-UC to aqueous systems to maximize its potential in many disciplines including artificial photosynthesis and biological studies such as bioimaging and photodynamic therapy. ${ }^{29-31}$ The study of TTA-UC in aqueous media has been mostly focused on water-dispersible microor nanoparticles coated with polymer shells, in which donors and acceptors were dissolved in the compartments of viscous organic solvents. ${ }^{29,30,32,33}$ However, the essential requirements for TTA-UC, i.e., triplet energy transfer and annihilation processes, rely on the molecular diffusion of TTAUC dyes (Figure S3, Supporting Information), which are inevitably suppressed in these viscous environments. ${ }^{32,33}$ The hydrophobic interior of aqueous micelles ${ }^{34}$ and lipid membranes ${ }^{35-38}$ has also been employed to dissolve hydrophobic TTA-UC dyes, where the similarly limited diffusion of chromophores in the interior of these molecular assemblies limits their performance.

To overcome these issues, we recently developed a synthetic, bola-type amphiphilic acceptor that self-assembles in water to give monolayer membranes. ${ }^{39}$ In this amphiphilic acceptor, a 9,10-diphenylanthracene (DPA) chromophore was covalently introduced in the middle of the molecule, so that it is aligned in the hydrophobic interior of the monolayer membranes. This allows the excited triplets to be kept away from the molecular oxygen dissolved in the bulk aqueous phase. As a result, the acceptor monolayer membrane showed TEM-based photon upconversion in aerated water with a moderate acceptor triplet lifetime of $238 \mu \mathrm{s}^{39}$ Meanwhile, the diversity and controllability of chromophore orientations in the bola-form monolayer membranes are generally inferior to those of the bilayer-type membranes. ${ }^{40,41}$ The bulky DPA chromophore occupies a large volume fraction of the amphiphile, which considerably enhances the rigidity of the monolayer membrane. As a result, high-temperature treatment under microwave irradiation was required to co-disperse anionic donor molecules in the hydrophobic moiety. Lacking in the previous aqueous self-assembled TTA-UC systems is the ability to easily accommodate TTA-UC chromophores, and the ability to rationally engineer the critical parameters, such as donor-to-acceptor triplet energy transfer efficiency, triplet lifetime, and low photoexcitation intensity. For this, simultaneous pursuit of adaptive accommodation properties for guest TTA-chromophores and the rigid microenvironment for suppressing thermal deactivation of the excited chromophores is required. A solution to this paradoxical issue would be to design molecular self-assemblies with reduced thermal vibrational energy which, at the same time, exert flexibility so that their molecular orientation is tuned upon binding of the guest molecules.

In this respect, we recently reported the formation of air-stable TTA-UC hydrogels by simply mixing TTA-UC chromophores with biopolymers (e.g. gelatin) and surfactants (e.g. Triton X-100). ${ }^{42}$ A relatively long triplet lifetime of $4.9 \mathrm{~ms}$ was observed in these quaternary biopolymer- surfactant donor-acceptor co-assembled gels; however, molecular-level information of those co-assembled structures in such a complex multicomponent mixture is difficult to obtain. Attaining a much longer triplet lifetime is essential to create superior TTA-UC systems that function under lower excitation light intensity, ${ }^{11-16,27,28}$ while there is no rational means to prolong the triplet lifetimes in molecular selfassemblies. Therefore, it is essential to understand the structure-function relationship by developing a much simpler and straightforward methodology that allows accumulation and alignment of TTA-UC chromophores in better-controlled aqueous assembly systems.

We herein show that a simple electrostatic complexation of anionic acceptors with suitably designed, cationic synthetic amphiphiles allows their densely packed self-assembly at the inner surface of bilayer membranes. This enables TEM-based TTA-UC at low excitation light intensity as low as the solar irradiance $\left(100 \mathrm{~mW} \mathrm{~cm}^{-2}\right.$, under AM1.5 conditions, for the whole solar spectrum ${ }^{15}$ ). Although the electrostatic adsorption of anionic cyanine dyes on the surface of cationic bilayer membranes and their singlet energy transfer characteristics have been reported, ${ }^{43}$ to avoid precipitation of the ion pairs, these hydrophobic dyes are introduced as a minor component and form patchy domains on the excessive bilayers. To attain the long-range triplet exciton migration among membranebound chromophores, the chromophores need to be regularly aligned on the whole membrane surface. For this, it is indispensable to secure the structural integrity of the ionpaired self-assemblies in water by ensuring hydrophilicity and cohesive interactions in the bilayers. ${ }^{11}$

9,10-Diphenylanthracene-2-sulfonate (DPAS) ${ }^{25}$ was employed in this work as an anionic acceptor (Figure 1). As DPAS is highly hydrophobic and practically insoluble in water, a cationic amphiphile $\mathbf{1}$ was designed to solubilize it as aqueous ion pairs. To alleviate the dehydration arising from the hydrophobic ion-pair formation, we introduced a hydroxyl group in the ammonium group and an amide-bond-enriched Lglutamate connector via a short alkyl chain spacer which are expected to enhance the hydration of the bilayer surface. The alkyl chain spacer moieties between the hydrophilic head groups and the double-chained glutamate groups provide hydrophobic binding sites that accommodate hydrophobic anionic dyes by flexibly changing the tilt angles of the spacer and the molecular orientation of the whole amphiphile. ${ }^{11,43,44}$ The multiple amide bonds and the ether linkages introduced near the L-glutamate connector show a flexible rearrangement of the hydrogen-bonding network structures and alkyl chain packing, which allow adaptation to the increased volume of ion-paired head groups while enhancing the structural stability of the self-assembly. ${ }^{44-46}$ Thus, amphiphile $\mathbf{1}$ is designed to maintain the integrity of ordered bilayer structures upon binding of hydrophobic DPAS to the membrane surface. As a result, the self-assembled ion pairs form bilayer membranes stably dispersed in water (Figure 1). Pt(II) 

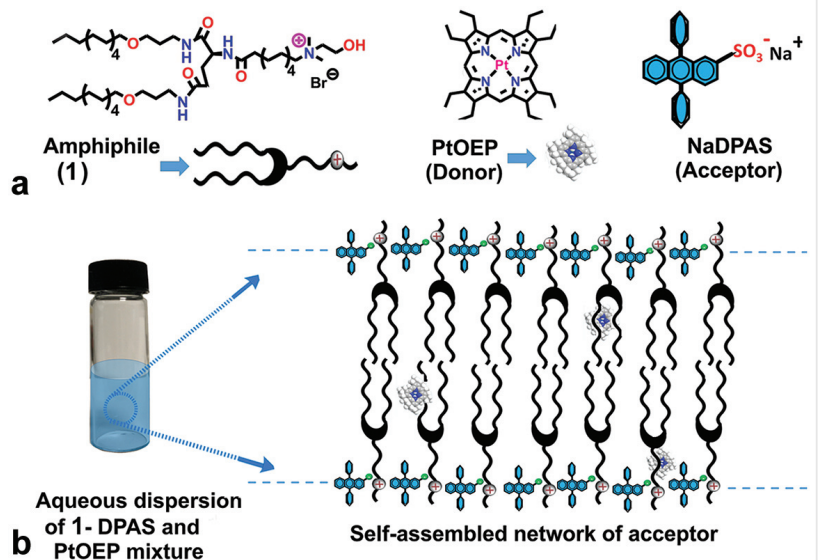

Figure 1 (a) Chemical structures of the cationic amphiphile, donor, and anionic acceptor. (b) Schematic illustration of the self-assembled ionpaired bilayer structure of $\mathbf{1}$ - DPAS in aqueous dispersion. PtOEP is dissolved in the hydrophobic interior of the ion-paired bilayer membrane.

octaethylporphyrin (PtOEP) was employed as a triplet sensitizer, which also was accommodated in the hydrophobic interior of the $\mathbf{1}$ - DPAS assembly. Photoexcitation of PtOEP molecules is followed by donor-to-acceptor triplet-triplet energy transfer (TTET), TEM among the acceptor arrays, and finally TTA and UC emission from the excited singlet states of the acceptor. Interestingly, a surprisingly long triplet lifetime of 8.0 ms was observed for 1-DPAS-PtOEP, which enabled TTA$\mathrm{UC}$ at low excitation intensity.

\section{Results and Discussion}

The synthesis of amphiphile $\mathbf{1}$ is reported in Scheme 1. The pristine amphiphile $\mathbf{1}$ is soluble in water and gave a homogeneous aqueous dispersion (Figure 2a). Meanwhile, the hydrophobic acceptor NaDPAS by alone was not soluble at the examined concentration $(4 \mathrm{mM})$. To prepare the amphiphile-chromophore complexes, amphiphile $\mathbf{1}$ and hydrophobic chromophores (DPAS and PtOEP) were mixed in a $\mathrm{CHCl}_{3}$ solution. After evaporating the solvent, the resulting residue was redispersed in Milli- $\mathrm{Q}$ water by heating and ultrasound sonication. These aqueous dispersions were then left to stand at room temperature for more than 1 hour before the measurement. The 2:1 mixture of $\mathbf{1}$ and NaDPAS in water (1-DPAS) gave a homogeneous translucent dispersion with enhanced light scattering (Figure $2 \mathrm{~b}$ ), which showed blue fluorescence under UV light (Figure 2c). This aqueous dispersion was stable, and no obvious phase separation or precipitation was observed for one week. The spectral and TTA-UC characteristics were obtained with good reproducibility as confirmed by a number of samples prepared separately. Thus, DPAS became stably dispersible in water by complexation with cationic bilayer membrane $\mathbf{1}$.

The self-assembled structure formed in aqueous 1 - DPAS was characterized by confocal laser scanning microscopy (CLSM), optical microscopy, transmission electron microscopy, differential scanning calorimetry (DSC), and absorption spectral measurements. A CLSM image showed fluorescence from developed fibrous objects, which overlapped well with a bright field image (Figure 2d-f). No phase separation between fluorescent DPAS and nonfluorescent $\mathbf{1}$ was observed. The in situ fluorescence spectra obtained by exciting self-assembled structures gave a fluorescence peak at around $440 \mathrm{~nm}$ (see Figure S4, Supporting Information), which matches the fluorescence peak of DPAS. ${ }^{25}$ In transmission electron microscopy, $\mathbf{1}$ formed aggregates of vesicle-like structures (Figure 2g), whereas plate-like structures were found for 1DPAS (Figure 2h). The observed change in morphology is consistent with the formation of the ion-paired bilayer membrane. ${ }^{11}$

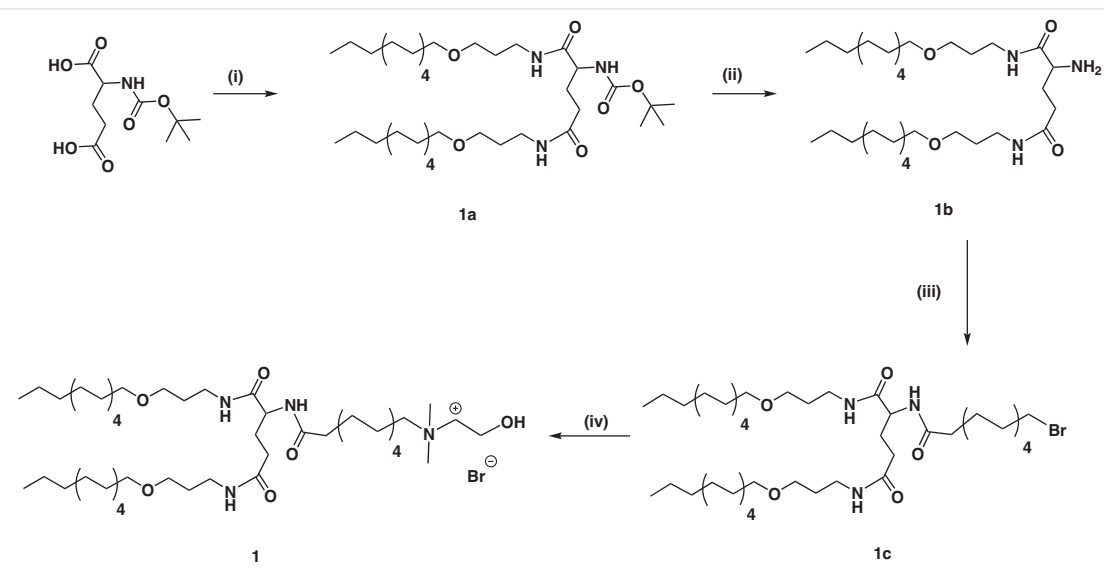

Scheme 1 Synthetic scheme for amphiphile 1: (i) 3-(dodecyloxy)propylamine, WSC, Et3N, HOBt, DCM, 12h, room temperature; (ii) TFA, DCM, room temperature, overnight; (iii) 1-bromoundecanoic acid, WSC, Et3N, HOBt, DCM, 12h, room temperature; (iv) 2-methylaminoethanol, CH3CN, reflux, 9h. Synthetic procedures are reported in References and Notes section. ${ }^{47}$ 


\begin{tabular}{|c|c|c|c|}
\hline & & THIEME & \\
\hline Organic Materials & D. Asthana et al. & $\begin{array}{l}\text { OPEN } \\
\text { ACCESS }\end{array}$ & Short Communication \\
\hline
\end{tabular}
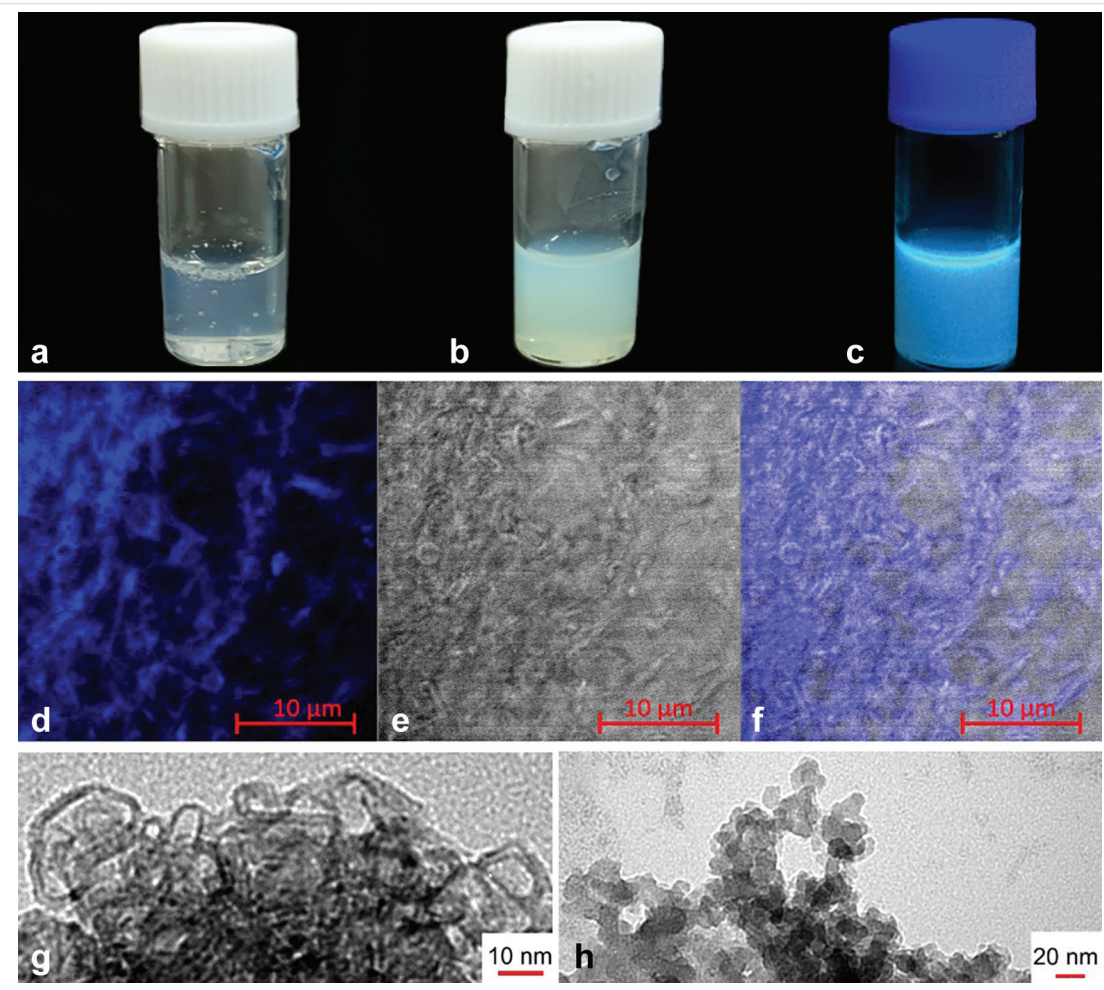

Figure 2 Pictures of (a) 1 and (b) 1-DPAS in water ([1] $=8 \mathrm{mM}$, [NaDPAS] $=4 \mathrm{mM})$. (c) A picture of 1-DPAS in water under UV light illumination. CLSM images of 1-DPAS in water showing (d) fluorescence image, (e) bright-field image, and (f) their overlap ( $\lambda_{\mathrm{ex}}=405 \mathrm{~nm}$ ). Transmission electron micrographs of specimens obtained from aqueous dispersions of $(\mathrm{g}) \mathbf{1}$ and $(\mathrm{h}) \mathbf{1}$-DPAS in water.

DSC investigations also showed changes in the gel-toliquid crystalline phase transition temperature (see Figure S5, Supporting Information). The aqueous dispersion of pristine amphiphile 1 showed broad gel-to-liquid crystalline transition peaks starting from $46{ }^{\circ} \mathrm{C}$, and the main transition peak was observed at $52.5^{\circ} \mathrm{C}$ (Figure S5a, Supporting Information). The broad pretransition is consistent with the bilayer membrane structures with multimodal hydrogen-bond networks and their thermal rearrangement. ${ }^{45}$ In contrast, the DSC thermogram for 1 - DPAS gives a sharp phase transition temperature $\left(T_{\mathrm{c}}\right)$ at $54.5{ }^{\circ} \mathrm{C}$ (Figure S5b, Supporting Information), which suggests enhanced cooperativity and stabilization of the gel phase due to the regular alignment of $\mathbf{1}$ - DPAS and an increase in the intermolecular interactions. By considering the stable water dispersibility of $\mathbf{1}$ - DPAS, it is natural to consider that the sulfonate group of the DPAS chromophore is ionpaired at the membrane surface, and the hydrophobic part is solubilized in the spacer-alkyl chain moiety of bilayer $\mathbf{1}$.

The electronic interactions between DPAS chromophores in 1-DPAS were further investigated by absorption, circular dichroism (CD), and circularly polarized luminescence (CPL) spectra. In the absorption spectra of aqueous 1DPAS, a structured ${ }^{1} \mathrm{~L}_{\mathrm{a}}$ band of anthracene chromophore was observed at around 325 to $430 \mathrm{~nm}$. Below the gel-toliquid crystalline phase transition temperature $\left(T_{\mathrm{c}}\right)$, the $0-0$ band is observed at $400 \mathrm{~nm}$. Upon heating the aqueous dispersion above $T_{\mathrm{c}}$, it showed a shift to $397.5 \mathrm{~nm}$ (Figure S6, Supporting Information). The observed spectral red-shift in the gel state reflects the presence of excitonic interactions among DPAS chromophores, as indicated by a couple of positive and negative cotton effects in the $C D$ spectrum (Figure S7a, Supporting Information). As DPAS is an achiral molecule, the molecular chirality of the glutamate moiety is transmitted to DPAS chromophores regularly aligned in the bilayer. ${ }^{48,49}$ Consistent with the observed excitonic coupling in the induced CD spectrum, CPL was observed with a reasonably high anisotropy factor of $g_{\text {lum }}=3.6 \times 10^{-2}$ at $470 \mathrm{~nm}$ (Figure S7b, Supporting Information), which is defined as $g_{\text {lum }}=2\left(I_{\mathrm{L}}-I_{\mathrm{R}}\right) /\left(I_{\mathrm{L}}+I_{\mathrm{R}}\right)$, where $I_{\mathrm{L}}$ and $I_{\mathrm{R}}$ are left- and right-circularly polarized emission intensities, respectively. ${ }^{50,51}$ These results confirm the ordered selfassembly of DPAS in the ion-paired aqueous bilayer membrane 1-DPAS.

We then scrutinized the TTA-UC properties for aqueous dispersions consisting of 1, DPAS, and PtOEP (1 - DPAS PtOEP, [1] $=8 \mathrm{mM},[$ DPAS $]=4 \mathrm{mM},[$ PtOEP $]=16 \mu \mathrm{M}$ ) under deaerated conditions (Figure 3a). The details of the optical setup are described in the Supporting Information. When excited by a 532-nm laser, upconverted emissions were observed for both of the deaerated and aerated 

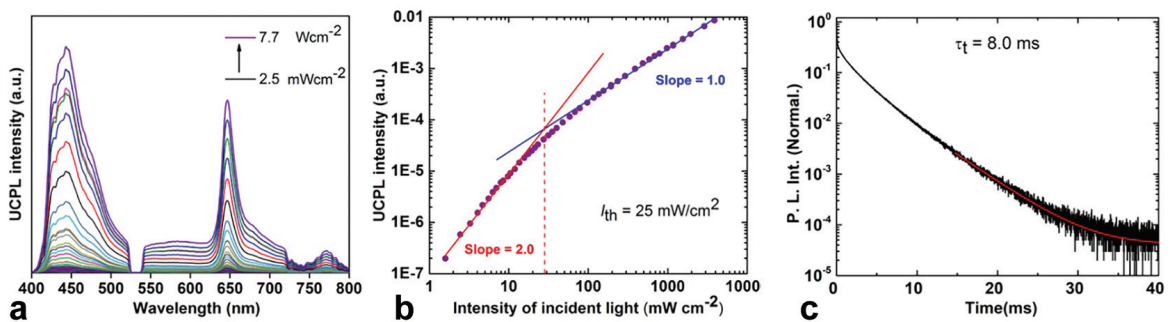

Figure 3 (a) Photoluminescence (PL) spectra of 1 - DPAS - PtOEP in deaerated water at room temperature under different excitation intensities $\left([1]=8 \mathrm{mM},[D P A S]=4 \mathrm{mM},[\right.$ PtOEP $]=16 \mu \mathrm{M}, \lambda_{\mathrm{ex}}=532 \mathrm{~nm}, 532 \mathrm{~nm}$ notch filter). (b) Excitation intensity dependence of UC PL intensity at $445 \mathrm{~nm}$. The linear fits with slopes of 2.0 and 1.0 in the lower and higher excitation intensity regimes are shown. (c) Excited state lifetime decay profile for 1 - DPAS - PtOEP in water $([1]=8 \mathrm{mM},[D P A S]=4 \mathrm{mM}$, [PtOEP $]=16 \mu \mathrm{M})$ The measurement was carried out in the deaerated condition at room temperature $\left(\lambda_{\mathrm{ex}}=531 \mathrm{~nm}, \lambda_{\mathrm{em}}=445 \mathrm{~nm}\right)$.

dispersions at around $432 \mathrm{~nm}$. Emission lifetime and excitation intensity dependence confirmed the TTA-based mechanism of the emission, as discussed below. Similar TTA-UC emission was observed in air-saturated water (Figure S8, Supporting Information), but its emission intensity was not stably maintained under continuous excitation. This would be ascribed to the diffusion of dissolved oxygen to DPAS chromophores assembled near the membrane surface. For better air stability, it is desirable to locate DPA chromophores in the deeper portion of bilayers beneath the hydrogen-bonding networks that show enhanced oxygen barrier properties. ${ }^{24,26,39,52}$ The UC quantum yield $\Phi_{\mathrm{UC}}$ ' observed for aqueous 1-DPAS-PtOEP was found to be $0.7 \%$ in the deaerated state (Figure S9, Supporting Information). In general, the quantum yield is defined as the ratio of absorbed photons to emitted photons, and thus the maximum yield $\left(\Phi_{\mathrm{UC}}\right)$ of the bimolecular TTAUC process is $50 \%$. However, this value is doubled in many reports to set the maximum quantum yield at $100 \%$. To avoid the confusion between these different definitions, the UC quantum yield is written as $\Phi_{\mathrm{UC}}{ }^{\prime}\left(=2 \Phi_{\mathrm{UC}}\right)$ when the maximum efficiency is normalized to be $100 \%$. The value of $\Phi_{\mathrm{UC}}{ }^{\prime}$ is proportional to the product of $f, \Phi_{\mathrm{ISC}}, \Phi_{\mathrm{ET}}, \Phi_{\mathrm{TTA}}$, and $\Phi_{\mathrm{FL}}$. The parameter $f$ is the statistical probability for obtaining a singlet excited state after the annihilation of two triplet states, and $\Phi_{\mathrm{ISC}}, \Phi_{\mathrm{ET}}, \Phi_{\mathrm{TTA}}$, and $\Phi_{\mathrm{FL}}$ represent the quantum efficiencies of donor ISC, donor-to-acceptor TTET, TTA between acceptor triplets and acceptor fluorescence. In the case of PtOEP, $\Phi_{\text {ISC }}$ is regarded as unity. $\Phi_{\mathrm{ET}}$ is determined by the ratio between the donor phosphorescence quantum yields with $\left(\Phi_{\mathrm{P}}\right)$ and without $\left(\Phi_{\mathrm{P}, 0}\right)$ acceptor $\left(\Phi_{\mathrm{ET}}=1-\Phi_{\mathrm{P}} / \Phi_{\mathrm{P}, 0}\right)$. The acceptor $\Phi_{\mathrm{FL}}$ was determined with an absolute quantum yield measurement system as described in the Supporting information.

In Figure 3a, residual phosphorescence intensity of PtOEP is observed at around $650 \mathrm{~nm}$, which is ascribable to those located in the hydrophobic interior of the ternary aqueous bilayer and not quenched by the surface acceptors. However, the ternary bilayer showed an overall high donor-to-acceptor triplet energy transfer efficiency $\Phi_{\mathrm{ET}}$ of ca. $93 \%$. Meanwhile, the fluorescence quantum yield of the acceptor $\left(\Phi_{\mathrm{FL}}\right)$ showed a decrease in the presence of the donor. The observed $\Phi_{\mathrm{FL}}(86 \%$ without the donor, $56 \%$ with the donor) would be ascribed to the singlet back energy transfer and reabsorption process (see the Supporting Information). We consider that the quenching of the upconverted fluorescence by the donor is a nonnegligible process contributing to the observed moderate upconversion quantum yield $\Phi_{\mathrm{Uc}}$.

To ensure the TEM-based UC (TEM-UC) in the present aqueous system, we measured the UC spectrum of 1 - DPAS - PtOEP in a frozen state $(77 \mathrm{~K})$. A UC emission with a vibrational structure was successfully observed, which confirms the contribution of the TEM-UC mechanism because the molecular diffusion-based mechanism is inhibited under these conditions (see Figure S10, Supporting Information). ${ }^{23-28}$

One of the important figures of merit for the TTA-UC system is a threshold excitation intensity $\left(I_{\mathrm{th}}\right)$ beyond which the TTA efficiency reaches its maximum. ${ }^{53,54}$ When the sunlight is used as an excitation source, it is desirable that the $I_{\text {th }}$ value is lower than the solar irradiance, i.e. 1.6 $\mathrm{mW} \mathrm{cm}^{-2}$ when PtOEP is employed as triplet sensitizers. ${ }^{24}$ The $I_{\text {th }}$ value is determined from the double logarithm plot of UC emission intensity against the incident light intensity, which shows a transition from the quadratic to the linear dependence (Figure 3b). ${ }^{15,53,54}$ This behavior is characteristic of the TTA-based UC, and the extrapolation of the two fitting lines gave a crossing point $I_{\text {th }}$ of $25 \mathrm{~mW} / \mathrm{cm}^{2}$. This $I_{\text {th }}$ value is still higher than the ideal value, but is remarkably low for TTA-UC in aqueous molecular assemblies, which underlines the superiority of the ion-paired aqueous bilayer system.

To elucidate factors contributing to the low $I_{\mathrm{th}}$ value, we measured the acceptor triplet lifetime $\tau_{\mathrm{T}}$. The acceptor triplet lifetime can be obtained from the decay time of the upconverted emission by the known relationship $I_{\mathrm{UC}}(t) \propto$ $\exp \left(-\mathrm{t} / \tau_{\mathrm{UC}}\right)=\exp \left(-2 t / \tau_{\mathrm{T}}\right)$ in the longer timescale region, when the annihilation efficiency becomes negligible compared with the spontaneous decay of the triplets. ${ }^{24,39}$ Considering the relationship of $I_{\mathrm{th}} \propto \tau_{\mathrm{T}}{ }^{-2}$, the triplet 
lifetime has the key to achieve TTA-UC under lowintensity incident light. ${ }^{53,54}$ However, the current methodology to engineer the triplet lifetime is still rudimentary. ${ }^{55,56}$ To our surprise, a first-order tail fit of the upconversion intensity decay profile from an aqueous dispersion of 1-DPAS-PtOEP gave a remarkably long triplet lifetime of $8.0 \mathrm{~ms}$ (Figure $3 \mathrm{c}$ ). To date, there has been no report of TTA-UC in aqueous systems with such a long triplet lifetime. This indicates that the deactivation of excited triplets is considerably suppressed in aqueous 1-DPAS-PtOEP, which would be consistent with the highdensity, regular alignment of DPAS chromophores in the multiply hydrogen-bonded bilayer. As shown by the DSC data (see Figure S5, Supporting Information), the electrostatic binding of DPAS at the inner surface of cationic bilayer 1 and the hydrogen-bond networks in the interior of 1-DPAS would have cooperatively enhanced the rigidity of the bilayer gel phase, which suppressed thermal deactivation of the excited triplets in the organized bilayer membranes.

\section{Conclusions}

In conclusion, we have demonstrated that the ion-pairbased strategy utilizing the multiple hydrogen bondforming cationic amphiphiles $\mathbf{1}$ and the anionic acceptor DPAS to achieve low-power TEM-UC in the aqueous medium. The maintenance of the total amphiphilicity is a key to stably disperse aqueous bilayers with DPAS counterions. The multiple hydrogen-bond networks formed in aqueous bilayers provide adaptive environments ${ }^{11,24}$ for the self-assembly of DPAS chromophores, and the rigid microenvironments of the acceptor-integrated bilayers lead to the observed lowest $I_{\text {th }}$ value among the existing aqueous TTAUC systems with a remarkably long triplet lifetime of $8.0 \mathrm{~ms}$. We envisage that the superior nature of the welldesigned ionic bilayer membranes would serve as platforms to self-organize excited triplets, which would lead to a diverse area of applications including bioimaging and theranostics.

\section{Funding Information}

This work was partly supported by JSPS KAKENHI Grant Numbers JP25220805, JP17H04799, JP16H06513 (Coordination Asymmetry), JP16H00844, JP14F04345.

\section{Acknowledgement}

D.A. acknowledges the JSPS postdoctoral fellowship for foreign researchers.

\section{Supporting Information}

Supporting information for this article is available online at https://doi.org/10.1055/s-0039-3400250.

\section{References and Notes}

(1) Rabinowitch, E., Govindjee. Photosynthesis. Wiley: New York, 1969.

(2) Blankenship, R. E. Molecular Mechanisms of Photosynthesis. Wiley-Blackwell: Hoboken, NJ, 2014.

(3) Kunitake, T.; Shimomura, M.; Hashiguchi, Y.; Kawanaka, T. J. Chem. Soc. Chem. Commun. 1985, 833.

(4) Tamai, N.; Yamazaki, T.; Yamazaki, I. Chem. Phys. Lett. 1988, 147, 25.

(5) Kimizuka, N.; Kunitake, T. J. Am. Chem. Soc. 1989, 11, 3758.

(6) Morita, T.; Kimura, S.; Imanishi, S. J. Am. Chem. Soc. 1999, 121, 581.

(7) Ajayaghosh, A.; George, S. J.; Praveen, V. K. Angew. Chem. Int. Ed. Engl. 2003, 42, 332.

(8) Kayser, V.; Turton, D. A.; Aggeli, A.; Beevers, A.; Reid, G. D.; Beddard, G. S. J. Am. Chem. Soc. 2004, 126, 336.

(9) Gao, M.; Paul, S.; Schwieters, C. D., et al. J. Phys. Chem. C Nanomater. Interfaces 2015, 119, 13948.

(10) Sethy, R.; Kumar, J.; Métivier, R., et al. Angew. Chem. Int. Ed. Engl. 2017, 56, 15053.

(11) Nakashima, T.; Kimizuka, N. Adv. Mater. 2002, 14, 1113.

(12) Baluschev, S.; Miteva, T.; Yakutkin, V.; Nelles, G.; Yasuda, A.; Wegner, G. Phys. Rev. Lett. 2006, 97, 143903.

(13) Singh-Rachford, T. N.; Castellano, F. N. Coord. Chem. Rev. 2010, $254,2560$.

(14) Zhao, J. Z.; Ji, S. M.; Guo, H. M. RSC Advances 2011, 1, 937.

(15) Monguzzi, A.; Tubino, R.; Hoseinkhani, S.; Campione, M.; Meinardi, F. Phys. Chem. Chem. Phys. 2012, 14, 4322.

(16) Simon, Y. C.; Weder, C. J. Mater. Chem. 2012, 22, 20817.

(17) Tayebjee, M. JY.; McCamey, D. R.; Schmidt, T. W. J. Phys. Chem. Lett. 2015, 6, 2367.

(18) Goldschmidt, J. C.; Fischer, S. Adv. Opt. Mater. 2015, 3, 510.

(19) Gray, V.; Moth-Poulsen, K.; Albinsson, B.; Abrahamsson, M. Coord. Chem. Rev. 2018, 362, 54.

(20) Lennartson, A.; Roffey, A.; Moth-Poulsen, K. Tetrahedron Lett. 2015, 56, 1457.

(21) Masutani, K.; Morikawa, M. A.; Kimizuka, N. Chem. Commun. (Camb.) 2014, 50, 15803.

(22) Ishiba, K.; Morikawa, M. A.; Chikara, C., et al. Angew. Chem. Int. Ed. Engl. 2015, 54, 1532.

(23) Duan, P.; Yanai, N.; Kimizuka, N. J. Am. Chem. Soc. 2013, 135, 19056.

(24) Duan, P.; Yanai, N.; Nagatomi, H.; Kimizuka, N. J. Am. Chem. Soc. 2015, 137, 1887.

(25) Hisamitsu, S.; Yanai, N.; Kimizuka, N. Angew. Chem. Int. Ed. Engl. 2015, 54, 11550.

(26) Ogawa, T.; Yanai, N.; Monguzzi, A.; Kimizuka, N. Sci. Rep. 2015, 5, 10882.

(27) Yanai, N.; Kimizuka, N. Chem. Commun. (Camb.) 2016, 52, 5354.

(28) Kimizuka, N.; Yanai, N.; Morikawa, M. A. Langmuir 2016, 32, 12304.

(29) Wohnhaas, C.; Turshatov, A.; Mailänder, V., et al. Macromol. Biosci. 2011, 11, 772.

(30) Wohnhaas, C.; Mailänder, V.; Dröge, M., et al. Macromol. Biosci. 2013, 13, 1422. 
(31) Zhou, J.; Liu, Q.; Feng, W.; Sun, Y.; Li, F. Chem. Rev. 2015, 115, 395.

(32) Liu, Q.; Yang, T.; Feng, W.; Li, F. J. Am. Chem. Soc. 2012, 134, 5390.

(33) Kim, J-H.; Kim, J-H. J. Am. Chem. Soc. 2012, 134, 17478.

(34) Turshatov, A.; Busko, D.; Baluschev, S.; Miteva, T.; Landfester, K. New J. Phys. 2011, 13, 083035.

(35) Askes, S. HC.; Bahreman, A.; Bonnet, S. Angew. Chem. Int. Ed. Engl. 2014, 53, 1029.

(36) Askes, S. HC.; López Mora, N.; Harkes, R., et al. Chem. Commun. (Camb.) 2015, 51, 9137.

(37) Poznik, V.; Faltermeier, V.; Dick, B.; König, B. RSC Advances 2016, 6, 41947.

(38) Askes, S. HC.; Brodie, P.; Bruylants, G.; Bonnet, S. J. Phys. Chem. B 2017, 121, 780.

(39) Kouno, H.; Ogawa, T.; Amemori, S.; Mahato, P.; Yanai, N.; Kimizuka, N. Chem. Sci. (Camb.) 2016, 7, 5224.

(40) Okahata, Y.; Kunitake, T. J. Am. Chem. Soc. 1979, 101, 5231.

(41) Fuhrhop, J. H.; Wang, T. Chem. Rev. 2004, 104, 2901.

(42) Bharmoria, P.; Hisamitsu, S.; Nagatomi, H., et al.J. Am. Chem. Soc. 2018, 140, 10848

(43) Nakashima, N.; Ando, R.; Kunitake, T. Bull. Chem. Soc. Jpn. 1987, 60, 1967.

(44) Asakuma, S.; Okada, H.; Kunitake, T. J. Am. Chem. Soc. 1991, 113, 1749.

(45) Kimizuka, N.; Kawasaki, T.; Kunitake, T. J. Am. Chem. Soc. 1993, $115,4387$.

(46) Kimizuka, N.; Kawasaki, T.; Hirata, K.; Kunitake, T. J. Am. Chem. Soc. 1998, 120, 4094.

(47) Synthesis of 2-amino-N1,N5-bis(3-(dodecyloxy)propyl)pentanediamide(1b): Boc-L-Glutamic acid $(2.83 \mathrm{~g}, 11.4 \mathrm{mmol}), 3-$ (dodecyloxy)propylamine $(6.2 \mathrm{~g}, 25.4 \mathrm{mmol}$ ) and triethyl amine $(4 \mathrm{~mL})$ were taken in a $500 \mathrm{~mL}$ round-bottomed flask and dissolved by adding $200 \mathrm{~mL}$ of dry dichloromethane (DCM). The mixture was stirred for 15 minutes under an ice bath. Subsequently, $\quad N$-(3-dimethylaminopropyl)- $N$ '-ethylcarbodiimide hydrochloride (WSC, $5.51 \mathrm{~g}, 28.7 \mathrm{mmol}$ ) and 1-hydroxybenzotriazole hydrate (HOBt, $4.4 \mathrm{~g}, 28.7 \mathrm{mmol}$ ) were dissolved in $100 \mathrm{~mL}$ dry DCM and added dropwise to the cooled reaction mixture. The reaction mixture was stirred for 12 hours under a nitrogen atmosphere with gradual heating to room temperature. Reaction progress was monitored by thin layer chromatography (TLC). When the reaction was complete, the solvent was evaporated and the crude was washed with $\mathrm{CHCl}_{3} /$ water for 3 times. The organic layer was dried over sodium sulfate and filtered. The solvent was evaporated to give a pale-yellow crude which was further purified by column chromatography (Silica, $\mathrm{HCl}_{3} / \mathrm{MeOH}$ ) to give a colorless solid product (1a, 75\% yield). The solid obtained in the first step (1a, $5.8 \mathrm{~g}, 8.5 \mathrm{mmol})$ was dissolved in dry DCM $(100 \mathrm{~mL})$ and treated with trifluoroacetic acid (TFA, $19 \mathrm{~g}, 167 \mathrm{mmol}$ ). The mixture was stirred overnight at room temperature. After reaction completion, DCM and TFA were evaporated under vacuum to give the product as a yellow oil which upon acidification with $35 \% \mathrm{HCl}$ (10 g) gave a colorless precipitate. The obtained precipitate was purified by recrystallization from the acetone/methanol solvent mixture (1b, $58 \%$ yield). ${ }^{1} \mathrm{H} \mathrm{NMR}\left(\mathrm{CDCl}_{3}, 300 \mathrm{MHz}\right): \delta(\mathrm{ppm})=8.61\left(\mathrm{~m}, 3 \mathrm{H}, \mathrm{NH}_{3}\right)$, $8.47(\mathrm{t}, 1 \mathrm{H}, \mathrm{CO}-\mathrm{NH}), 7.42(\mathrm{t}, 1 \mathrm{H}, \mathrm{CO}-\mathrm{NH}), 4.30(\mathrm{~m}, 1 \mathrm{H}$, tert.-CH), 3.51-3.01 (m, 12H), $2.60(\mathrm{~m}, 2 \mathrm{H}), 2.25(\mathrm{~m}, 2 \mathrm{H}), 1.95-1.68(\mathrm{~m}, 4 \mathrm{H})$, 1.66-1.02 (m, 40H), $0.90\left(\mathrm{t}, 6 \mathrm{H}, \mathrm{CH}_{3}\right)$. (Figure S1, Supporting Information).

Synthesis of 11-((18,22-dioxo-13,27-dioxa-17,23-diazanonatriacontan-19-yl)amino)-N-(2-hydroxyethyl)-N,N-dimethyl11-oxoundecan-1-aminium (1): The deprotected intermediate product from the previous step (1) $1.6 \mathrm{~g}, 2.7 \mathrm{mmol}$ ) was taken in $60 \mathrm{~mL}$ dry DCM and $\mathrm{Et}_{3} \mathrm{~N}$ ( $5 \mathrm{mmol}$ ) followed by addition of 11-bromo-undecanoic acid $(0.82 \mathrm{~g}, 3.1 \mathrm{mmol})$ and DMAP $(0.045 \mathrm{~g}, 0.37 \mathrm{mmol})$. The reaction mixture was stirred under ice-bath. A WSC $(0.6 \mathrm{~g}, 3.1 \mathrm{mmol})$ solution in $20 \mathrm{~mL}$ dry DCM was added dropwise and stirring was continued for 12 hours at room temperature. After completion of the reaction, the solvent was evaporated, and a residue was washed by $\mathrm{CHCl}_{3} /$ water mixture. The organic phase was dried over sodium sulfate and filtered. The solvent was evaporated under vacuum, and crude was purified by recrystallization from acetone (1c, 78\% yield). Finally, intermediate $1 \mathrm{c}(0.9 \mathrm{~g}, 1.0 \mathrm{mmol})$ was reacted with 2-methylaminoethanol $(0.5 \mathrm{~g}, 5.5 \mathrm{mmol})$ in acetonitrile under reflux condition. The mixture was stirred for 9 hours at $80^{\circ} \mathrm{C}$. The solvent was evaporated to yield white crude which was purified by re-precipitation from $\mathrm{MeOH}$ solution by adding $\mathrm{CH}_{3} \mathrm{CN}$ (45\% yield). ${ }^{1} \mathrm{H}-\mathrm{NMR}(\mathrm{CDCl} 3,300 \mathrm{MHz}): \delta(\mathrm{ppm})=\delta$ $(\mathrm{ppm})=7.41(\mathrm{~d}, 1 \mathrm{H}, \mathrm{CO}-\mathrm{NH}), 7.30(\mathrm{t}, 1 \mathrm{H}, \mathrm{CO}-\mathrm{NH}), 6.8(\mathrm{t}, 1 \mathrm{H}, \mathrm{CO}-$ $\mathrm{NH}), 4.37(\mathrm{~m}, 1 \mathrm{H}$, tert.-CH), $4.1(\mathrm{~m}, 2 \mathrm{H}), 3.73(\mathrm{~m}, 2 \mathrm{H}), 3.22-3.59$ (m, 20H), 2.32-2.42 (m, 4H), 1.10-2.10 (m, 64H), $0.88\left(\mathrm{t}, 6 \mathrm{H}, \mathrm{CH}_{3}\right)$. (Figure S2, Supporting Information). MALDI-TOF MS spectrum (dithranol matrix): Calculated molecular weight $\mathrm{C}_{50} \mathrm{H}_{101} \mathrm{~N}_{4} \mathrm{O}_{6}{ }^{+}$ $=854.36$; Obtained $=854.4$. Elemental analysis calculated for $\mathrm{C}_{50} \mathrm{H}_{101} \mathrm{~N}_{4} \mathrm{O}_{6} \mathrm{Br}+\mathrm{H}_{2} \mathrm{O}$ : C, 63.06; $\mathrm{H}, 10.90 ; \mathrm{Br}, 8.39 ; \mathrm{N}, 5.88 ; \mathrm{O}$, 11.76. Found: C, 63.34; H, 11.04; N, 5.98.

(48) Nakashima, N.; Fukushima, H.; Kunitake, T. Chem. Lett. 1981, 10 , 1207.

(49) Kawasaki, T.; Tokuhiro, M.; Kimizuka, N.; Kunitake, T. J. Am. Chem. Soc. 2001, 123, 6792.

(50) Okazaki, Y.; Goto, T.; Sakaguchi, R., et al. Chem. Lett. 2016, 45, 448.

(51) Kumar, J.; Nakashima, T.; Kawai, T. J. Phys. Chem. Lett. 2015, 6, 3445.

(52) Overton, E. Vierteljahrsschr. Naturf. Ges. Zürich 1899, 44, 88.

(53) Widomska, J.; Raguz, M.; Subczynski, W. K. Biochim. Biophys. Acta. 2007, 1768, 2635.

(54) Monguzzi, A.; Mezyk, J.; Scotognella, F.; Tubino, R.; Meinardi, F. Phys. Rev. B Condens. Matter Mater. Phys. 2008, 78, 195112.

(55) Hirata, S.; Totani, K.; Zhang, J., et al. Adv. Funct. Mater. 2013, 23, 3386.

(56) An, Z.; Zheng, C.; Tao, Y., et al. Nat. Mater. 2015, 14, 685. 